\title{
New bijections from $n$-color compositions
}

\author{
Caroline Shapcott
}

\begin{abstract}
Combinatorial bijections are given from the set of $n$-color compositions of $\nu$, for which a part of size $n$ can take on $n$ colors, to the set of compositions of $2 \nu-1$ having only parts of size 1 or 2 , the set of compositions of $2 \nu$ having only odd parts, and the set of compositions of $2 \nu+1$ having no parts of size 1 . A generalized bijection based on similar ideas is then given between the set of compositions of $\nu$ into parts congruent to $a(\bmod b)$ and the set of compositions of $\nu+b-a$ into parts congruent to $b(\bmod a)$ with each part greater than $b-a$.
\end{abstract}

AMS 2000 SUBJeCt Classifications: Primary 05A19 (Combinatorial identities, bijective combinatorics), 11B39 (Fibonacci and Lucas numbers).

KEYWORDS AND PHRASES: Integer compositions, restricted compositions, $n$-color compositions, Fibonacci numbers.

\section{Introduction}

A composition of $\nu$ is a sequence of positive integers called parts that sum to $\nu$. An $n$-color composition of $\nu$ is a composition of $\nu$ for which a part of size $n$ can take on $n$ colors. It has been shown using generating functions that the number of $n$-color compositions of $\nu$ is $F_{2 \nu}$, the $2 \nu$-th Fibonacci number [1]. For example:

\begin{tabular}{|c|c|c|cc|}
\hline$\nu=1$ & $\nu=2$ & $\nu=3$ & \multicolumn{2}{|c|}{$\nu=4$} \\
\hline$\left(1_{1}\right)$ & $\left(2_{1}\right)$ & $\left(3_{1}\right)$ & $\left(4_{1}\right)$ & $\left(2_{1}, 2_{1}\right)$ \\
& $\left(2_{2}\right)$ & $\left(3_{2}\right)$ & $\left(4_{2}\right)$ & $\left(2_{2}, 2_{1}\right)$ \\
& $\left(1_{1}, 1_{1}\right)$ & $\left(3_{3}\right)$ & $\left(4_{3}\right)$ & $\left(2_{1}, 2_{2}\right)$ \\
& & $\left(2_{1}, 1_{1}\right)$ & $\left(4_{4}\right)$ & $\left(2_{2}, 2_{2}\right)$ \\
& & $\left(2_{2}, 1_{1}\right)$ & $\left(3_{1}, 1_{1}\right)$ & $\left(2_{1}, 1_{1}, 1_{1}\right)$ \\
& & $\left(1_{1}, 2_{1}\right)$ & $\left(3_{2}, 1_{1}\right)$ & $\left(2_{2}, 1_{1}, 1_{1}\right)$ \\
& & $\left(1_{1}, 2_{2}\right)$ & $\left(3_{3}, 1_{1}\right)$ & $\left(1_{1}, 2_{1}, 1_{1}\right)$ \\
& & $\left(1_{1}, 1_{1}, 1_{1}\right)$ & $\left(1_{1}, 3_{1}\right)$ & $\left(1_{1}, 2_{2}, 1_{1}\right)$ \\
& & & $\left(1_{1}, 3_{2}\right)$ & $\left(1_{1}, 1_{1}, 2_{1}\right)$ \\
& & & $\left(1_{1}, 3_{3}\right)$ & $\left(1_{1}, 1_{1}, 2_{2}\right)$ \\
& & & \multicolumn{2}{|c|}{$\left(1_{1}, 1_{1}, 1_{1}, 1_{1}\right)$} \\
\hline$F_{2}=1$ & $F_{4}=3$ & $F_{6}=8$ & \multicolumn{2}{|c}{$F_{8}=21$} \\
\hline
\end{tabular}


The following relationships between restricted compositions and Fibonacci numbers are also well-known (see [3], for example): The number of compositions of $\nu$ having only parts of size 1 or 2 , referred to here as 1 - 2 compositions, is $F_{\nu+1}$. The number of compositions of $\nu$ having only odd parts, referred to here as odd compositions, is $F_{\nu}$. The number of compositions of $\nu$ having no parts of size 1 , referred to here as 1 -free compositions, is $F_{\nu-1}$. Combinatorial bijections are therefore anticipated, for appropriately shifted values of $\nu$, between these sets and the set of $n$-color compositions; however, to the author's knowledge and according to Heubach and Mansour's recent comprehensive text on compositions [3, p. 88], no such bijection has yet been demonstrated.

In this paper, we represent $n$-color compositions symbolically using strings of crosses $(x)$ and dashes $(-)$. The symbolic representation provides an unexpected and immediate structural link to 1-2 compositions. We then perform transformations on the character strings in order to obtain odd and 1-free compositions. Finally, we apply an adaptation of those transformations in order to answer the following open problem posed in a recent paper by Diffenderfer [2]: Give a combinatorial bijection between the set of compositions of $\nu$ into parts congruent to $a(\bmod b)$ and the set of compositions of $\nu+b-a$ into parts congruent to $b(\bmod a)$ with each part greater than $b-a$.

Proposition 1. Let $\lambda=\left(\lambda_{1}^{c_{1}}, \ldots, \lambda_{t}^{c_{t}}\right)$ denote an $n$-color composition of $\nu$ with $t$ parts, where $\lambda_{i}^{c_{i}}$ is a part of size $\lambda_{i}$ with color $1 \leq c_{i} \leq \lambda_{i}$. Let $p=\left(p_{1}, \ldots, p_{2 t-1}\right)$ denote a list of the positions of the crosses in a string of $\nu+t-1$ characters of which $2 t-1$ are crosses and $\nu-t$ are dashes. The set of all such $\lambda s$ is in one-to-one correspondence with the set of all such ps.

Proof. The symbolic representation of an $n$-color composition is achieved as follows: A part of size $\lambda_{i}$ with color $1 \leq c_{i} \leq \lambda_{i}$ is represented by a string of $\lambda_{i}$ characters, all of which are dashes except for the $c_{i}$-th character which is a cross. The full representation is then formed by concatenating these character strings (in order) and separating adjacent strings by an additional cross. For example, the $n$-color compositions of 3 are represented as follows:

\begin{tabular}{|c|c|}
\hline$n$-color comps(3) & cross-and-dash \\
\hline$\left(3_{1}\right)$ & $\mathrm{x}--$ \\
$\left(3_{2}\right)$ & $-\mathrm{x}-$ \\
$\left(3_{3}\right)$ & $--\mathrm{x}$ \\
$\left(2_{1}, 1_{1}\right)$ & $\mathrm{x}-\mathrm{x} \times$ \\
$\left(2_{2}, 1_{1}\right)$ & $-\mathrm{x} \times \mathrm{x}$ \\
$\left(1_{1}, 2_{1}\right)$ & $\mathrm{x} \times \mathrm{x}-$ \\
$\left(1_{1}, 2_{2}\right)$ & $\mathrm{x} \times-\mathrm{x}$ \\
$\left(1_{1}, 1_{1}, 1_{1}\right)$ & $\mathrm{x} \times \mathrm{x} \times \mathrm{x}$ \\
\hline
\end{tabular}


Note that an $n$-color composition of $\nu$ with $t$ parts corresponds to exactly one string of $\nu+t-1$ characters of which $2 t-1$ are crosses and $\nu-t$ are dashes: The number of crosses is $t+(t-1)=2 t-1$, one for each part and one between each consecutive part; the number of dashes is $\nu-t$, the sum of all the part sizes minus $t$ for the single crosses marking the color of each part. We will occasionally refer to the cross-and-dash representation as a list of characters, $s=\left(s_{1}, \ldots, s_{\nu+t-1}\right)$.

Formally, given any $n$-color composition $\lambda=\left(\lambda_{1}^{c_{1}}, \ldots, \lambda_{t}^{c_{t}}\right)$ of $\nu$, build a list $p=\left(p_{1}, \ldots, p_{2 t-1}\right)$ of the positions of the crosses in a string of $\nu+t-1$ characters by $T(\lambda)=p$, where $p_{i}$ is given by

$$
\begin{cases}p_{1}=c_{1} & 1 \leq i \leq t-1 \\ p_{2 i}=\sum_{k=1}^{i}\left(\lambda_{k}+1\right) & 1 \leq i \leq t-1 \\ p_{2 i+1}=\sum_{k=1}^{i}\left(\lambda_{k}+1\right)+c_{i+1}\end{cases}
$$

Given any list $p=\left(p_{1}, \ldots, p_{2 t-1}\right)$ of the positions of the crosses in a string of $\nu+t-1$ characters, build an $n$-color composition $\lambda=\left(\lambda_{1}^{c_{1}}, \ldots, \lambda_{t}^{c_{t}}\right)$ of $\nu$ by $T^{*}(p)=\lambda$, where $\lambda_{i}$ and $c_{i}$ are given by

$$
\begin{cases}c_{1}=p_{1} & \\ c_{i}=p_{2 i-1}-p_{2 i-2} & 2 \leq i \leq t \\ \lambda_{1}=p_{2}-1 & \\ \lambda_{i}=p_{2 i}-p_{2 i-2}-1 & 2 \leq i \leq t-1 \\ \lambda_{t}=\nu+t-1-p_{2 t-2} . & \end{cases}
$$

Note that, using the above equations, it can be verified directly that $T \circ$ $T^{*}(p)=p$ and $T^{*} \circ T(\lambda)=\lambda$. For example, the $2 i$-th position in $T \circ T^{*}(p)$ is

$$
\sum_{k=1}^{i}\left(\lambda_{k}+1\right)=\left(\lambda_{1}+1\right)+\sum_{k=2}^{i}\left(\lambda_{k}+1\right)=p_{2}+\sum_{k=2}^{i}\left(p_{2 k}-p_{2 k-2}\right)=p_{2 i}
$$

and the $i$-th position in $T^{*} \circ T(\lambda)$ is

$$
p_{2 i}-p_{2 i-2}-1=\sum_{k=1}^{i}\left(\lambda_{k}+1\right)-\sum_{k=1}^{i-1}\left(\lambda_{k}+1\right)-1=\lambda_{i}
$$

with color

$$
p_{2 i-1}-p_{2 i-2}=\sum_{k=1}^{i-1}\left(\lambda_{k}+1\right)+c_{i}-\sum_{k=1}^{i-1}\left(\lambda_{k}+1\right)=c_{i} .
$$


Similar calculations can be made in both directions for the remaining positions.

\section{1-2 compositions}

Proposition 2. Let $\lambda=\left(\lambda_{1}^{c_{1}}, \ldots, \lambda_{t}^{c_{t}}\right)$ denote an $n$-color composition of $\nu$ with $t$ parts, where $\lambda_{i}^{c_{i}}$ is a part of size $\lambda_{i}$ with color $1 \leq c_{i} \leq \lambda_{i}$. Let $\omega=\left(\omega_{1}, \ldots, \omega_{\nu+t-1}\right)$ denote a 1-2 composition of $2 \nu-1$ with $\nu+t-1$ parts. The set of all such $\lambda s$ is in one-to-one correspondence with the set of all such $\omega$ s.

Proof. First write the cross-and-dash representation of an $n$-color composition. Then simply let crosses represent parts of size 1 and let dashes represent parts of size 2. For example:

\begin{tabular}{|c|c|c|}
\hline$n$-color comps(3) & cross-and-dash & 1 -2 comps $(5)$ \\
\hline$\left(3_{1}\right)$ & $\mathrm{x}--$ & $(1,2,2)$ \\
$\left(3_{2}\right)$ & $-\mathrm{x}-$ & $(2,1,2)$ \\
$\left(3_{3}\right)$ & $--\mathrm{x}$ & $(2,2,1)$ \\
$\left(2_{1}, 1_{1}\right)$ & $\mathrm{x}-\mathrm{x} \times$ & $(1,2,1,1)$ \\
$\left(2_{2}, 1_{1}\right)$ & $-\mathrm{x} \times \mathrm{x}$ & $(2,1,1,1)$ \\
$\left(1_{1}, 2_{1}\right)$ & $\mathrm{x} \times \mathrm{x}-$ & $(1,1,1,2)$ \\
$\left(1_{1}, 2_{2}\right)$ & $\mathrm{x} \times-\mathrm{x}$ & $(1,1,2,1)$ \\
$\left(1_{1}, 1_{1}, 1_{1}\right)$ & $\mathrm{x} \times \mathrm{x} \times \mathrm{x}$ & $(1,1,1,1,1)$ \\
\hline
\end{tabular}

Formally, given any $n$-color composition of $\nu$ and its unique cross-anddash representation $s=\left(s_{1}, \ldots, s_{\nu+t-1}\right)$ (guaranteed by Proposition 1), build a 1-2 composition $\omega=\left(\omega_{1}, \ldots, \omega_{\nu+t-1}\right)$ of $2 \nu-1$ by defining

$$
\omega_{i}= \begin{cases}1 & \text { if } s_{i}=\mathrm{x} \\ 2 & \text { if } s_{i}=-\end{cases}
$$

This construction is clearly reversible.

We are guaranteed to create compositions of $2 \nu-1$ in this manner since the number of crosses is $2 t-1$ and the number of dashes is $\nu-t$. Therefore, since crosses represent parts of size 1 and dashes represent parts of size 2, an $n$-color composition of $\nu$ with $t$ parts corresponds to a 1-2 composition of

$$
1 \cdot(2 t-1)+2 \cdot(\nu-t)=2 \nu-1 .
$$




\section{Odd compositions}

Proposition 3. Let $\lambda=\left(\lambda_{1}^{c_{1}}, \ldots, \lambda_{t}^{c_{t}}\right)$ denote an n-color composition of $\nu$ with $t$ parts, where $\lambda_{i}^{c_{i}}$ is a part of size $\lambda_{i}$ with color $1 \leq c_{i} \leq \lambda_{i}$. Let $\omega=\left(\omega_{1}, \ldots, \omega_{2 t}\right)$ denote an odd composition of $2 \nu$ with $2 t$ parts. The set of all such $\lambda s$ is in one-to-one correspondence with the set of all such $\omega s$.

Proof. Beginning with the cross-and-dash representation, we now view crosses as separators. To the left and right of every cross, replace a string of $j$ dashes with a string of $2 j+1$ dashes, which then represents a part of size $2 j+1$. Note that all part sizes are inherently odd. For example:

\begin{tabular}{|c|c|c|c|}
\hline$n$-color comps(3) & cross-and-dash & dashes as parts & odd comps(6) \\
\hline$\left(3_{1}\right)$ & $\mathrm{x}--$ & $-\mathrm{x}-----$ & $(1,5)$ \\
$\left(3_{2}\right)$ & $-\mathrm{x}-$ & $---\mathrm{x}---$ & $(3,3)$ \\
$\left(3_{3}\right)$ & $--\mathrm{x}$ & $-----\mathrm{x}-$ & $(5,1)$ \\
$\left(2_{1}, 1_{1}\right)$ & $\mathrm{x}-\mathrm{x} \mathrm{x}$ & $-\mathrm{x}---\mathrm{x}-\mathrm{x}-$ & $(1,3,1,1)$ \\
$\left(2_{2}, 1_{1}\right)$ & $-\mathrm{x} \times \mathrm{x}$ & $---\mathrm{x}-\mathrm{x}-\mathrm{x}-$ & $(3,1,1,1)$ \\
$\left(1_{1}, 2_{1}\right)$ & $\mathrm{x} \times \mathrm{x}-$ & $-\mathrm{x}-\mathrm{x}-\mathrm{x}---$ & $(1,1,1,3)$ \\
$\left(1_{1}, 2_{2}\right)$ & $\mathrm{x} \mathrm{x}-\mathrm{x}$ & $-\mathrm{x}-\mathrm{x}---\mathrm{x}-$ & $(1,1,3,1)$ \\
$\left(1_{1}, 1_{1}, 1_{1}\right)$ & $\mathrm{x} \times \mathrm{x} \times \mathrm{x}$ & $-\mathrm{x}-\mathrm{x}-\mathrm{x}-\mathrm{x}-\mathrm{x}-$ & $(1,1,1,1,1,1)$ \\
\hline
\end{tabular}

Formally, given any $n$-color composition of $\nu$ and its unique cross-anddash representation (guaranteed by Proposition 1), make the following transformation. To the left and right of every cross, replace a string of $j$ dashes with a string of $2 j+1$ dashes $(0 \leq j \leq \nu-t)$, and let $\ell_{i}$ be the length of the $i$-th string of dashes $(i=1, \ldots, 2 t)$. Then build an odd composition $\omega=\left(\omega_{1}, \ldots, \omega_{2 t}\right)$ of $2 \nu$ by defining $\omega_{i}=\ell_{i}$. This construction is easily reversed by replacing each string of $j$ dashes with a string of $\frac{j-1}{2}$ dashes $(1 \leq j \leq 2 \nu-2 t+1)$.

We are guaranteed to create compositions of $2 \nu$ in this manner by the following reasoning: Suppose a given $n$-color composition has $t$ parts and that its cross-and-dash representation has $\beta_{i}$ strings of $i$ consecutive dashes. Then the new number of dashes in the cross-and-dash transformation is

$$
\sum_{i=1}^{\nu}(2 i+1) \beta_{i} .
$$

Since $\sum_{i=1}^{\nu} i \beta_{i}=\nu-t$ (the original number of dashes) and $\sum_{i=1}^{\nu} \beta_{i}=$ $(2 t-1)+1=2 t$ (one more than the number of crosses), we are constructing 
compositions of

$$
\sum_{i=1}^{\nu}(2 i+1) \beta_{i}=2 \sum_{i=1}^{\nu} i \beta_{i}+\sum_{i=1}^{\nu} \beta_{i}=2(\nu-t)+2 t=2 \nu .
$$

\section{1-free compositions}

Proposition 4. Let $\lambda=\left(\lambda_{1}^{c_{1}}, \ldots, \lambda_{t}^{c_{t}}\right)$ denote an $n$-color composition of $\nu$ with $t$ parts, where $\lambda_{i}^{c_{i}}$ is a part of size $\lambda_{i}$ with color $1 \leq c_{i} \leq \lambda_{i}$. Let $\omega=\left(\omega_{1}, \ldots, \omega_{\nu-t+1}\right)$ denote a 1-2 composition of $2 \nu+1$ with $\nu-t+1$ parts. The set of all such $\lambda s$ is in one-to-one correspondence with the set of all such $\omega s$.

Proof. Beginning with the cross-and-dash representation, we now view dashes as separators. To the left and right of every dash, replace a string of $j$ crosses with a string of $j+2$ crosses, which then represents a part of size $j+2$. Note that all part sizes are inherently greater than 1. For example:

\begin{tabular}{|c|c|c|c|}
\hline$n$-color comps(3) & cross-and-dash & crosses as parts & 1-free comps(7) \\
\hline$\left(3_{1}\right)$ & $\mathrm{x}--$ & $\mathrm{x} \times \mathrm{x}-\mathrm{x} \times \mathrm{x} \times \mathrm{x}$ & $(3,2,2)$ \\
$\left(3_{2}\right)$ & $-\mathrm{x}-$ & $\mathrm{x} \times \mathrm{x} \times \mathrm{x} \times \mathrm{x} \times$ & $(2,3,2)$ \\
$\left(3_{3}\right)$ & $--\mathrm{x}$ & $\mathrm{x} \times \mathrm{x} \times \mathrm{x}-\mathrm{x} \times \mathrm{x}$ & $(2,2,3)$ \\
$\left(2_{1}, 1_{1}\right)$ & $\mathrm{x}-\mathrm{x} \times$ & $\mathrm{x} \times \mathrm{x}-\mathrm{x} \times \mathrm{x} \times$ & $(3,4)$ \\
$\left(2_{2}, 1_{1}\right)$ & $-\mathrm{x} \times \mathrm{x}$ & $\mathrm{x} \times \mathrm{x} \times \mathrm{x} \times \mathrm{x}$ & $(2,5)$ \\
$\left(1_{1}, 2_{1}\right)$ & $\mathrm{x} \times \mathrm{x}-$ & $\mathrm{x} \times \mathrm{x} \times \mathrm{x}-\mathrm{x} \times$ & $(5,2)$ \\
$\left(1_{1}, 2_{2}\right)$ & $\mathrm{x} \times \mathrm{x}$ & $\mathrm{x} \times \mathrm{x} \times \mathrm{x} \times \mathrm{x} \times$ & $(4,3)$ \\
$\left(1_{1}, 1_{1}, 1_{1}\right)$ & $\mathrm{x} \times \mathrm{x} \times \mathrm{x}$ & $\mathrm{x} \times \mathrm{x} \times \mathrm{x} \times \mathrm{x}$ & $(7)$ \\
\hline
\end{tabular}

Formally, given any $n$-color composition of $\nu$ and its unique cross-anddash representation (guaranteed by Proposition 1), make the following transformation. To the left and right of every dash, replace a string of $j$ crosses with a string of $j+2$ crosses $(0 \leq j \leq 2 t-1)$, and let $\ell_{i}$ be the length of the $i$-th string of crosses $(i=1, \ldots, \nu-t+1)$. Then build a 1 -free composition $\omega=\left(\omega_{1}, \ldots, \omega_{\nu-t+1}\right)$ of $\nu$ by defining $\omega_{i}=\ell_{i}$. This construction is easily reversed by replacing each string of $j$ crosses with a string of $j-2$ crosses $(2 \leq j \leq 2 t+1)$.

We are guaranteed to create compositions of $2 \nu+1$ in this manner by the following reasoning: Suppose a given $n$-color composition has $t$ parts, and its cross-and-dash representation has $\gamma_{i}$ strings of $i$ crosses. Then the new number of crosses in the cross-and-dash transformation is

$$
\sum_{i=1}^{\nu}(i+2) \gamma_{i}
$$


Since $\sum_{i=1}^{\nu} i \gamma_{i}=2 t-1$ (the original number of crosses) and $\sum_{i=1}^{\nu} \gamma_{i}=$ $(\nu-t)+1=\nu-t+1$ (one more than the number of dashes), we are constructing compositions of

$$
\sum_{i=1}^{\nu}(i+2) \gamma_{i}=\sum_{i=1}^{\nu} i \gamma_{i}+2 \sum_{i=1}^{\nu} \gamma_{i}=(2 t-1)+2(\nu-t+1)=2 \nu+1 .
$$

\section{Generalized bijection}

While this section is not directly related to $n$-color compositions, it employs the ideas of Propositions 3 and 4 in order to construct a generalized bijective proof of the following theorem by Diffenderfer and Sills. The theorem is proven in [2], while background is given in [4].

Theorem 1. Suppose $a \leq b$. The number of compositions of $\nu$ into parts congruent to $a(\bmod b)$ equals the number of compositions of $\nu+b-a$ into parts congruent to $b(\bmod a)$ where each part is greater than $b-a$.

Bijective proof. The essence of the proof is as follows: Begin with a composition of $\nu$ into parts congruent to $a(\bmod b)$. Construct a string of crosses and dashes using the ideas of Proposition 3, i.e. the parts of the composition are represented by strings of dashes, and adjacent parts are separated by crosses. Make a transformation on the strings of dashes by replacing a string of $j$ dashes with a string of $\frac{j-a}{b}$ dashes. Make a second transformation on the strings of crosses by replacing a string of $j$ crosses with a string of $a j+b$ crosses. Extract a composition of $\nu+b-a$, whose parts are congruent to $b(\bmod a)$ and greater than $b-a$, by using the ideas of Proposition 4 , i.e. strings of crosses represent the parts of the composition, and dashes separate adjacent parts. The steps are easily reversed by replacing a string of $j$ crosses with a string of $\frac{j-b}{a}$ crosses, then replacing a string of $j$ dashes with a string of $b j+a$ dashes.

We provide a guiding example with $\nu=10, a=2$, and $b=3$ :

\begin{tabular}{|c|c|c|c|c|}
\hline comps(10) & dashes as parts & & crosses as parts & comps(11) \\
\hline$(8,2)$ & $--------\times--$ & $--\times$ & $\times \times \times-\times \times \times-\times \times \times \times \times$ & $(3,3,5)$ \\
$(5,5)$ & $-----\times-----$ & $-\times-$ & $\times \times \times-\times \times \times \times \times-\times \times \times$ & $(3,5,3)$ \\
$(2,8)$ & $--\times--------$ & $\times--$ & $\times \times \times \times \times-\times \times \times-\times \times \times$ & $(5,3,3)$ \\
$(2,2,2,2,2)$ & $--\times--\times--\times--\times--$ & $\times \times \times \times$ & $\times \times \times \times \times \times \times \times \times \times \times$ & $(11)$ \\
\hline
\end{tabular}

The left column contains compositions of $\nu=10$ with parts congruent to $a(\bmod b)=2(\bmod 3)$. The right column contains compositions of $\nu+b-a=$ 11 with parts congruent to $b(\bmod a)=3(\bmod 2)$, i.e. $1(\bmod 2)$, and greater than $b-a=1$. 
Formally, there are five steps in the bijection, which we will label $T_{1}$ through $T_{5}$, and five inverses, which we will label $T_{1}^{*}$ through $T_{5}^{*}$. The main ideas having been outlined above, we now describe each step in detail. We use the composition $(2,8)$ from the table above as a guiding example, referenced in brackets after each step.

- $T_{1}(\lambda)=p$

Given a composition $\lambda=\left(\lambda_{1}, \ldots, \lambda_{t}\right)$ of $\nu$ with $t$ parts, all of which are congruent to $a(\bmod b)$, build a cross-and-dash representation by concatenating strings of dashes of length $\lambda_{i}$, each separated by a cross. There are $t-1$ crosses and $\nu$ dashes. Record a list $p=\left(p_{1}, \ldots, p_{t-1}\right)$ of the positions of the crosses, where $p_{i}$ is given by

$$
p_{i}=\sum_{k=1}^{i}\left(\lambda_{k}+1\right)
$$

[For the composition $(2,8)$, we have $t-1=1$ cross and $\nu=10$ dashes. We compute $p_{1}=\lambda_{1}+1=3$ and record $p=(3)$ for the position of the cross.]

- $T_{2}(p)=\hat{p}$

Replace a string of $j$ dashes with a string of $\frac{j-a}{b}$ dashes. Since $j=$ $\lambda_{i} \equiv a(\bmod b)$, we have $\frac{j-a}{b} \in \mathbb{Z}_{+}$. Note that some crosses may now be adjacent. There are now $t-1$ crosses and $\frac{\nu-a t}{b}$ dashes, by the following reasoning: Let $\beta_{i}$ be the number of strings of dashes of length $i$. Since $\sum_{i=1}^{\nu} i \beta_{i}=\nu$ (the original number of dashes) and $\sum_{i=1}^{\nu} \beta_{i}=t$ (one more than the number of crosses), the new number of dashes is

$$
\sum_{i=1}^{\nu} \beta_{i}\left(\frac{i-a}{b}\right)=\frac{1}{b} \sum_{i=1}^{\nu} i \beta_{i}-\frac{a}{b} \sum_{i=1}^{\nu} \beta_{i}=\frac{\nu-a t}{b}
$$

Due to the first term in the equation above and the fact that $\frac{j-a}{b} \in$ $\mathbb{Z}_{+}$, we have $\frac{\nu-a t}{b} \in \mathbb{Z}_{+}$. Record a list $\hat{p}=\left(\hat{p}_{1}, \ldots, \hat{p}_{t-1}\right)$ of the new positions of the crosses, where $\hat{p}_{i}$ is given by

$$
\hat{p}_{i}=\frac{p_{i}-i-a i}{b}+i
$$

[We now have $t-1=1$ cross and $\frac{\nu-a t}{b}=2$ dashes. We compute $\hat{p}_{1}=\frac{p_{1}-1-a \cdot 1}{b}+1=1$ and record $\hat{p}=(1)$ for the new position of the cross.] 
- $T_{3}(\hat{p})=\hat{q}$

Take the complement of $\hat{p}$ to build a list $\hat{q}=\left(\hat{q}_{1}, \ldots, \hat{q}_{\frac{\nu-a t}{b}}\right)$ of the positions of the dashes. In other words, begin with a list of all positive integers in the interval $[1, d]$, where $d=t-1+\frac{\nu-a t}{b}$ is the current total number of crosses and dashes, and remove all integers that occur in $\hat{p}$. [Since $d=3$, we record $\hat{q}=(2,3)$ for the positions of the dashes.]

- $T_{4}(\hat{q})=q$

Replace a string of $j$ crosses with a string of $a j+b$ crosses. The new lengths of all strings of crosses are now inherently congruent to $b(\bmod a)$. Moreover, they are at least length $b$ (or equivalently, greater than length $b-a$ ). There are now $\nu+b-a$ crosses and $\frac{\nu-a t}{b}$ dashes, by the following reasoning: Let $\gamma_{i}$ be the number of strings of crosses of length $i$. Since $\sum_{i=0}^{\nu} i \gamma_{i}=t-1$ (the original number of crosses) and $\sum_{i=0}^{\nu} \gamma_{i}=\frac{\nu-a t}{b}+1$ (one more than the number of dashes), the new number of crosses is

$$
\sum_{i=0}^{\nu} \gamma_{i}(a i+b)=a \sum_{i=0}^{\nu} i \gamma_{i}+b \sum_{i=0}^{\nu} \gamma_{i}=\nu+b-a .
$$

Record a list $q=\left(q_{1}, \ldots, q_{\frac{\nu-a t}{b}}\right)$ of the new positions of the dashes, where $q_{i}$ is given by

$$
q_{i}=\left(\hat{q}_{i}-i\right) a+b i+i .
$$

[We now have $\nu+b-a=11$ crosses and $\frac{\nu-a t}{b}=2$ dashes. We compute $q_{1}=\left(\hat{q}_{1}-1\right) a+b \cdot 1+1=6$ and $q_{2}=\left(\hat{q}_{2}-2\right) a+b \cdot 2+2=10$. We record $q=(6,10)$ for the new positions of the dashes.]

- $T_{5}(q)=\omega$

Build a composition of $\nu+b-a$ with $\frac{\nu-a t}{b}+1$ parts by concatenating the lengths of the strings of crosses. This composition has parts congruent to $b(\bmod a)$, all of which are at least $b$. The composition is $\omega=\left(\omega_{1}, \ldots, \omega_{\frac{\nu-a t}{b}+1}\right)$, where $\omega_{i}$ is given by

$$
\left\{\begin{array}{l}
\omega_{1}=q_{1}-1 \\
\omega_{i}=q_{i}-q_{i-1}-1 \\
\omega_{\frac{\nu-a t}{b}+1}=c-q_{\frac{\nu-a t}{b}}
\end{array} \quad 2 \leq i \leq \frac{\nu-a t}{b}\right.
$$

and $c=\nu+b-a+\frac{\nu-a t}{b}$ is the current total number of crosses and dashes. 
[Since $\frac{\nu-a t}{b}+1=3$, we build a composition with 3 parts. We compute $\omega_{1}=q_{1}-1=5$ and $\omega_{2}=q_{2}-q_{1}-1=3$ and $\omega_{3}=c-q_{2}=3$. We record $\omega=(5,3,3)$ as the new composition of $\nu+b-a=11$.]

- $T_{5}^{*}(\omega)=q$

Given a composition $\omega=\left(\omega_{1}, \ldots, \omega_{\frac{\nu-a t}{b}+1}\right)$ of $\nu+b-a$ with $\frac{\nu-a t}{b}+1$ parts, all of which are congruent to $b(\bmod a)$ and greater than $b-$ $a$, build a cross-and-dash representation by concatenating strings of crosses of length $\omega_{i}$, each separated by a dash. There are $\nu+b-a$ crosses and $\frac{\nu-a t}{b}$ dashes. Record a list $q=\left(q_{1}, \ldots, q_{\frac{\nu-a t}{b}}\right)$ of the positions of the dashes, where $q_{i}$ is given by

$$
q_{i}=\sum_{k=1}^{i}\left(\omega_{k}+1\right)
$$

[We have $\nu+b-a=11$ crosses and $\frac{\nu-a t}{b}=2$ dashes. We compute $q_{1}=\omega_{1}+1=6$ and $q_{2}=\omega_{1}+1+\omega_{2}+1=10$. We record $q=(6,10)$ as the positions of the dashes.]

- $T_{4}^{*}(q)=\hat{q}$

Replace a string of $j$ crosses with a string of $\frac{j-b}{a}$ crosses. Since $j=$ $\omega_{i} \equiv b(\bmod a)$, we have $\frac{j-b}{a} \in \mathbb{Z}_{+}$. Note that some dashes may now be adjacent. There are now $t-1$ crosses and $\frac{\nu-a t}{b}$ dashes, by the following reasoning: Let $\gamma_{i}$ be the number of strings of crosses of length $i$. Since $\sum_{i=1}^{\nu} i \gamma_{i}=\nu+b-a$ (the original number of crosses) and $\sum_{i=1}^{\nu} \gamma_{i}=\frac{\nu-a t}{b}+1$ (one more than the number of dashes), the new number of crosses is

$$
\sum_{i=1}^{\nu} \gamma_{i}\left(\frac{i-b}{a}\right)=\frac{1}{a} \sum_{i=1}^{\nu} i \gamma_{i}-\frac{b}{a} \sum_{i=1}^{\nu} \gamma_{i}=t-1 .
$$

Record a list $\hat{q}=\left(\hat{q}_{1}, \ldots, \hat{q}_{\frac{\nu-a t}{b}}\right)$ of the new positions of the dashes, where $\hat{q}_{i}$ is given by

$$
\hat{q}_{i}=\frac{q_{i}-i-b i}{a}+i \text {. }
$$

[We now have $t-1=1$ cross and $\frac{\nu-a t}{b}=2$ dashes. We compute $\hat{q}_{1}=\frac{q_{1}-1-b \cdot 1}{a}+1=2$ and $\hat{q}_{2}=\frac{q_{2}-2-b \cdot 2}{a}+2=3$. We record $\hat{q}=(2,3)$ as the new positions of the dashes.]

- $T_{3}^{*}(\hat{q})=\hat{p}$

Take the complement of $\hat{q}$ to build a list $\hat{p}=\left(\hat{p}_{1}, \ldots, \hat{p}_{t-1}\right)$ of the positions of the crosses. In other words, begin with a list of all positive 
integers in the interval $[1, d]$, where $d=t-1+\frac{\nu-a t}{b}$ is the current total number of crosses and dashes, and remove all integers that occur in $\hat{q}$. [Since $d=3$, we record $\hat{p}=(1)$ as the position of the cross.]

- $T_{2}^{*}(\hat{p})=p$

Replace a string of $j$ dashes with a string of $b j+a$ dashes. The new lengths of all strings of dashes are now inherently congruent to $a(\bmod b)$. (They are also inherently at least length $a$, although this does not affect the outcome since it is implied that a part congruent to $a(\bmod b)$ cannot be smaller than $a$.) There are now $t-1$ crosses and $\nu$ dashes, by the following reasoning: Let $\beta_{i}$ be the number of strings of dashes of length $i$. Since $\sum_{i=0}^{\nu} i \beta_{i}=\frac{\nu-a t}{b}$ (the original number of dashes) and $\sum_{i=0}^{\nu} \beta_{i}=t$ (one more than the number of crosses), the new number of dashes is

$$
\sum_{i=0}^{\nu} \beta_{i}(b i+a)=b \sum_{i=0}^{\nu} i \beta_{i}+a \sum_{i=0}^{\nu} \beta_{i}=\nu .
$$

Record a list $p=\left(p_{1}, \ldots, p_{t-1}\right)$ of the new positions of the crosses, where $p_{i}$ is given by

$$
p_{i}=\left(\hat{p}_{i}-i\right) b+a i+i .
$$

[We now have $t-1=1$ cross and $\nu=10$ dashes. We compute $p_{1}=$ $\left(\hat{p}_{1}-1\right) b+a \cdot 1+1=3$. We record $p=(3)$ as the new position of the cross.]

- $T_{1}^{*}(p)=\lambda$

Build a composition of $\nu$ with $t$ parts by concatenating the lengths of the strings of dashes. This composition has parts congruent to $a(\bmod b)$, all of which are at least $a$. The composition is $\lambda=\left(\lambda_{1}, \ldots, \lambda_{t}\right)$, where $\lambda_{i}$ is given by

$$
\left\{\begin{array}{l}
\lambda_{1}=p_{1}-1 \\
\lambda_{i}=p_{i}-p_{i-1}-1 \quad 2 \leq i \leq t-1 \\
\lambda_{t}=c^{*}-p_{t-1}
\end{array}\right.
$$

and $c^{*}=\nu+t-1$ is the current total number of crosses and dashes. [Since $t=2$, we build a composition with 2 parts. We compute $\lambda_{1}=$ $p_{1}-1=2$ and $\lambda_{2}=c^{*}-p_{1}=8$. We record $\lambda=(2,8)$ as the new composition of $\nu=10$.]

Each transformation can be checked to verify that $T_{1} \circ T_{1}^{*}(p)=p, T_{1}^{*} \circ$ $T_{1}(\lambda)=\lambda$, and so forth for $T_{2}$ through $T_{5}$. 


\section{Remarks}

While Propositions 2, 3, and 4 describe only the paths from $n$-color compositions to 1-2, odd, and 1-free compositions, the same transformations can be used to describe paths between the three sets of restricted compositions. The following example shows a path from 1-free compositions of 6 to odd compositions of 5 :

\begin{tabular}{|c|c|c|c|c|}
\hline $\operatorname{comps}(6)$ & crosses as parts & & dashes as parts & $\operatorname{comps}(5)$ \\
\hline$(6)$ & $x \times x \times x \times$ & $x \times x \times$ & $-x-x-x-x-$ & $(1,1,1,1,1)$ \\
\hline$(4,2)$ & $x \times x x-x x$ & $x x-$ & $-x-x---$ & $(1,1,3)$ \\
\hline$(3,3)$ & $x \times x-x \times x$ & $x-x$ & $-x---x-$ & $(1,3,1)$ \\
\hline$(2,4)$ & $x x-x \times x x$ & $-x x$ & $---x-x-$ & $(3,1,1)$ \\
\hline$(2,2,2)$ & $x x-x x-x x$ & -- & ----- & $(5)$ \\
\hline
\end{tabular}

The parts of the 1-free compositions are represented with strings of crosses, each separated by a dash, as shown in the second column in the table above. A string of $j$ crosses is then replaced with a string of $j-2$ crosses, resulting in the third column. A string of $j$ dashes is then replaced with a string of $2 j+1$ dashes, resulting in the fourth column. (Note that during this step, a string of 0 dashes will result in a single dash.) Then the parts of the odd compositions are represented by strings of dashes, each separated by a cross. As in the proof of Theorem 1, the character strings in the center column do not correspond with $n$-color compositions; they are merely stepping stones in the character transformation.

\section{References}

[1] A. K. Agarwal. n-colour compositions. Indian J. Pure Appl. Math., 31(11):1421-1427, 2000. MR1808110

[2] J. Diffenderfer. A bijection between two classes of restricted compositions. Fibonacci Quart., 50(4):360-365, 2012. MR3004612

[3] S. Heubach and T. Mansour. Combinatorics of Compositions and Words. Discrete Mathematics and its Applications (Boca Raton). CRC Press, Boca Raton, FL, 2010. MR2531482

[4] A. V. Sills. Compositions, partitions, and Fibonacci numbers. Fibonacci Quart., 49(4):348-354, 2011. MR2852008 
Caroline Shapcott

Indiana University South Bend

South Bend, Indiana

USA

E-mail address: shapcott@iusb.edu

Received January 6, 2013 